\title{
Ambiguity in Partnerships ${ }^{12}$
}

\author{
by
}

\section{David Kelsey and Willy Spanjers ${ }^{3}$}

\begin{abstract}
The consequences of ambiguity for partnerships are addressed. Partnerships with symmetric linear production functions are analysed in a Choquet Expected Utility (CEU)framework. Nash equilibrium in pure strategies is extended to CEU-games. A class of sharing rules are proposed that make constructive use of strategic ambiguity in partnerships. Results on ex-ante efficiency and ex-post efficiency of the outcomes are presented.
\end{abstract}

Keywords: Partnerships, Ambiguity, Choquet Expected Utility, Entrepreneur. JEL Classification Codes: D20, D23, D81, D82.

\section{INTRODUCTION}

1.1. Motivation. Our motivation for this paper comes from two observations. Firstly, economic theory predicts that it should be difficult to sustain cooperation due to free riding and moral hazard problems. Yet in practice, there seems to be considerably more cooperation than the theoretical prediction suggests. For example, consider the Lifeboat institute, which manages fast and efficient rescue at sea, despite relying on voluntary contributions to pay for lifeboats and volunteers to man them. There are many other examples. Experimental evidence shows a similar story. In experiments, subjects are much more cooperative than conventional economic theory would predict. ${ }^{4}$

The second observation is that in many economic situations probabilities are not given. Moreover it is not an easy task to assign meaningful probabilities to the relevant events. Formally we refer to situations in which probabilities are poorly defined as ambiguous. In the presence of ambiguity people tend to behave cautiously. In particular, they place more weight on bad outcomes than an expected utility maximiser would. We call this pessimism or ambiguity aversion.

\footnotetext{
${ }^{1}$ Address for correspondence: David Kelsey, Department of Economics, The University of Birmingham, Edgbaston, Birmingham B15 2TT, United Kingdom. E-mail: D.Kelsey@bham.ac.uk

${ }^{2}$ Research supported by ESRC grant no. R000222597, by the British Academy and by the Commerzbankstiftung. We would like to thank Jürgen Eichberger, John Fender, Shasikanta Nandeibam, Peter Sinclair, participants of ESEM'97 in Toulouse, the Royal Economic Society '98 conference in Coventry, the Second International Conference on Public Economic Theory 2000 in Coventry and the First World Conference of the Game Theory Society 2000 in Bilbao, as well as three anonymous referees and three editors for helpful comments.

${ }^{3}$ Department of Economics, The University of Birmingham, Edgbaston, Birmingham B15 2TT, United Kingdom.

${ }^{4}$ See e.g. Camerer (1997), Davis and Holt (1993, Ch. 5) and Roth (1995).
} 
We relate these two observations by showing that pessimism can be exploited to sustain cooperation in partnerships with ambiguous actions. Ambiguity can make threats more effective. The argument suggests that ambiguous threats can be exploited to sustain cooperation.

1.2. Evidence. There have been many experiments on choice under uncertainty. ${ }^{5}$ The strongest conclusions which can be obtained from this literature are as follows.

1. Compared to Savage's subjective expected utility theory, people over-weight unlikely events and under-weight likely events, ${ }^{6}$

2. The less familiar the source of uncertainty, the lower the weights given to all events, the less the over-weighting of unlikely events and the greater the under-weighting of likely events. ${ }^{7}$

The psychological intuition behind this is that people are relatively insensitive to changes in probability, other than changes between possibility and impossibility. Thus an increase in probability from 0 to $5 \%$ is given much greater weight than an increase from 50 to $55 \%$. As subjective expected utility is linear in probabilities, it predicts the two changes should be equivalent.

Our model aims to incorporate the key features of this evidence. We have focused on the under-weighting of large probabilities and the effects of familiarity of source, which will cause individuals to behave pessimistically. Under-weighting produces preferences which place more emphasis on bad outcomes. The "missing" probability mass tends to be assigned to bad outcomes rather than to specific events. For analytic simplicity we have abstracted from the over-weighting of small events.

There is less evidence on the effect of ambiguity in games. The available evidence seems loosely supportive of our argument. Consider the ultimatum bargaining game. As is well known, player 1 will offer player 2 approximately half the pie in experiments on this game. This is in contrast to the sub-game perfect equilibrium, in which player 1 keeps virtually all of

\footnotetext{
${ }^{5}$ See e.g. Ellsberg (1961) and Camerer and Weber (1992).

${ }^{6}$ See Gonzalez and Wu (1999).

${ }^{7}$ See Kilka and Weber (1998).
} 
the pie for himself. The dictator and impunity games are similar to the ultimatum bargaining, except that player 1 is completely protected against retaliation. ${ }^{8}$ In such games player 1 tends to behave in selfish ways, which are quite close to the predictions of economic models. Thus player 1 cooperates when player 2 has an ambiguous threat. When the ambiguity is removed cooperation ceases.

Public reactions to environmental scares show similar patterns to the laboratory evidence discussed above. Consider the public reactions to BSE, which has never been a major cause of death or illness, even compared to other sources of contamination in food. Yet it provoked a widespread scare, even in countries with minimal exposure to BSE. It does not seem unreasonable to argue that this reaction was due to ambiguity surrounding a new disease. In some cases, the panic was greater in countries less exposed to the disease. It is highly suggestive that the panic was caused by ambiguity, as in those countries the risk was less familiar. This appears to show a similar pattern to the laboratory evidence discussed above. A likely but unfamiliar event, that BSE does not pose a significant risk to the vast majority of people, is under-weighted. The shortfall is assigned to the bad outcome.

1.3. Outline of the Paper. A brief account of the background of our model is provided in Section 2. We both discuss basic models of partnerships and the ideas of Knight (1921) concerning ambiguity and entrepreneurs. The basic intuition of our model, which combines both strands, is outlined. Additional examples are discussed for which its logic applies. In Section 3 our representation of ambiguity is described. We generalize the concept of Nash equilibrium in pure strategies for this framework. The model of a partnership is presented in Section 4 and the entrepreneurial sharing rule is introduced.

In Section 5 this sharing rule is illustrated by a simple example. Conditions under which any equilibrium is ex-post or ex-ante efficient are stated in Section 6. The ex-post efficient outcome can be implemented, even when the partners have identical beliefs about the (aggregate) behaviour of the others. In Section 7 concluding remarks are made. We briefly discuss some broader questions our approach may raise. These concern the role of rationality, ambiguity in

\footnotetext{
${ }^{8}$ See Bolton and Zwick (1995), Forsythe, Horowitz, Savin and Sefton (1994) and Hofman, McCabe, Shacat and Smith (1994).
} 
a dynamic framework and optimism. Most technical material is gathered in the Appendix.

\section{PARTNERSHIPS}

2.1. The Role of Partnerships. Questions concerning optimal contracts for sharing the profits of partnerships are interesting both from a theoretical and from an applied perspective. From the practical point of view, partnerships play a role in everyday life in the ways lawyers, accountants, management consultants and doctors organize their firms. They can even be found in cooperation treaties of coalition governments, in multi-national organizations and in financial systems. From the theoretical point of view, two aspects are prominent. On the one hand, there is the problem of mechanism design, focusing on the implementation of efficient outcomes in groups of agents. In this context, the problem is that some individual characteristics of agents are private information. Agents may have an incentive to mimic the optimal behaviour of agents with different characteristics. An example is the free rider problem in the provision of public goods.

On the other hand, there is a moral hazard problem when the actions of the different partners fail to be observable or it is not possible to write an enforceable contract on them. As is usual in the literature on partnerships, we focus on this moral hazard problem with multiple agents that arises within the partnership. The individual characteristics of the agents are assumed to be common knowledge.

2.2. Implementing the Efficient Outcome. The analysis focuses on the kind of partnerships that are most problematic. These are partnerships where the agents are identical and the production function is linear and symmetric. When some shirking takes place, it is impossible to identify the shirker or even a strict subset of agents that contains the shirker(s). ${ }^{9}$

When the partnership can commit to destroying part of the production, there is an easy way to implement the efficient outcome. A contract can be used, that assigns each agent an equal share of the produced good if the efficient production level is obtained. Otherwise no payment is made. But it may be difficult to commit to actually destroying the output if the

\footnotetext{
${ }^{9}$ If in the case of unilateral shirking it is possible to identify some team within the partnership that contains the (unique) shirker, then it may be possible to implement the efficient outcome. This is discussed in Nandeibam (1997).
} 
target is missed. The agents have an incentive to renegotiate and divide the produced goods between them. Some agents may anticipate this and choose to shirk, trusting to end up with a part of the output without facing the corresponding cost of effort.

Alternatively, the following contract may be considered. One member of the partnership is designated to be the budget breaker, who does not produce. Each other agent obtains an equal share of the produced good if the efficient production level is reached. Whenever the total production is smaller, the budget breaker obtains the output and the other agents get nothing. Thus, it allows the latter in a clever way to "destroy" production. This solution is in the spirit of Holmström (1982).

The solution has two drawbacks. Firstly, as observed by Haller (1985), it falls short of implementing the efficient outcome since the "budget breaker" does not produce. For large partnerships, however, the per agent shortfall from the efficient payoff is small. Secondly, as Eswaran and Kotwal (1984) argue, the budget breaker may form a coalition with some other agent. They may agree that this agent is to shirk to ensure the budget breaker obtains the total production of the others. The proceeds are then divided between them. Andolfatto and Nosal (1997), however, note that such coalition may fail to form. After the agent shirked, the budget breaker has an incentive to renege on the promised side-payment. ${ }^{10}$

2.3. Partnership Puzzles. The intuition captured in the above gives rise to some puzzles. Certain partnerships are organized in a way that should lead to more shirking instead of less.

One class of examples are entrepreneur owned firms. In such firms the entrepreneur hires workers and pays their wages. Otherwise he is the residual claimant of the profits. In countries with rigid redundancy laws, the workers do not face an immediate threat of dismissal when they shirk. Theory suggests this should not provide good incentives. By the same token, less shirking should be expected in small collectively owned enterprises. Casual observation suggests that in reality the opposite is true. We propose that the presence of ambiguity may explain such puzzles.

In entrepreneur owned firms, workers face ambiguity about the effort provided by the

\footnotetext{
${ }^{10}$ For other solutions to the problem see, e.g., Rasmusen (1987), Legros and Matsushima (1991) and d'Asperment and Gérard-Varet (1998).
} 
entrepreneur. An entrepreneur is a specialist in dealing with the uncertainties of the market, e.g., in recognizing new opportunities for profit. Employees typically lack the entrepreneurial skills of judgement. So even when workers have a fair idea about the time an entrepreneur spends in his firm, this fails to reveal the quality of the entrepreneurial input. Therefore, it does not help them to resolve their ambiguity about the entrepreneur's effort.

2.4. Entrepreneurs and Ambiguity. The role of entrepreneurs in the organization of production when ambiguity prevails is discussed extensively in Knight (1921, Chapter IX). Regarding the role of entrepreneurs he is of the opinion that ${ }^{11}$

...there must come into play the diversity among men in degree of confidence in their judgement and powers and disposition to act upon their opinion, to "venture"...

...the confident and venturesome "assume" the risk or "insure" the doubtful and timid by guaranteeing the latter a specified income in return for an assignment of the actual results. ${ }^{12}$

In Knight's view, entrepreneurs are people who are more willing to bear ambiguity. This could be because they have better powers of judgement and experience less ambiguity in a given situation, or because they are less averse to it. This is confirmed by research. It provides three different types of explanations why entrepreneurs may be overly confident or optimistic. The first suggests that the more confident self-select to be entrepreneurs. ${ }^{13}$ In an evolutionary process irrational overconfident entrepreneurs may persist. ${ }^{14}$ Alternatively, it has been shown that it may be in the interest of firm owners to hire overconfident optimist managers. As the agency costs for such managers are less than for rational managers, overconfident optimists may be hired, even when they are less capable. ${ }^{15}$

Another aspect of the organization of production mentioned by Knight (1921, p. 356) is that

\footnotetext{
${ }^{11}$ Knight (1921, pp. 269-270).

${ }^{12}$ It seems, although the text reads "risk", it is "ambiguity" that is referred to.

${ }^{13}$ This is the basic idea of Rigotti and Ryan (1997). They argue entrepreneurs self-select to be less influenced by the sources of ambiguity.

${ }^{14}$ See Bernardo and Welch (2001).

${ }^{15}$ See Gervais, Heaton and Odean (2000).
} 
....specialized skills and training. .. are acquired in connection with and for use in the particular business. The cost of acquisition is borne chiefly by the worker and if the business is unprofitable, the loss generally falls on him.

We propose a class of sharing rules for partnerships with strategic ambiguity, which to some extent incorporate these properties. We call them "entrepreneurial sharing rules". One of the partners is assigned a position similar to that of a Knightian entrepreneur. He insures the income of the others, as long as it cannot be proven that they all shirked. If, however, it can be proven that each of the other partners did shirk, then they pay him a fine. This fine can, e.g., be a reimbursement for the loss of investment in partnership specific capital.

2.5. The Intuition. In a two person partnership with an entrepreneurial sharing rule, the following may happen. With some confidence, the worker correctly expects the entrepreneur to put in the agreed effort. But due to ambiguity, he does not completely rule out that the latter may shirk. Should the entrepreneur fail to provide any effort, shirking by the worker can be proven and he is fined for it. If the worker does not shirk, he is paid his wage, independent of the effort of the entrepreneur. Whenever he faces sufficient ambiguity that the entrepreneur provides the agreed effort, the worker refrains from shirking. The reason is that ambiguity increases the weight he places on the bad outcome, i.e., on being fined.

Suppose, in contrast, the entrepreneur is sufficiently confident that the worker does not shirk. As a consequence, he has to pay the worker's wage and obtains the output of the partnership. The best he can do is to choose his efficient effort level. As a result, neither the worker nor the entrepreneur shirks and the efficient outcome is implemented.

2.6. Other Examples. The logic of entrepreneurial sharing rules and the results we obtain apply more generally than to entrepreneur-owned firms. Other applications can be found in the realm of international politics. Firstly, consider military alliances as NATO or the first Gulf war coalition against Iraq. One common feature is, that shirking of a small number of its members had the potential to end in an unfavourable outcome. Another feature is the strong involvement and leadership of the USA, who effectively acted as a political residual claimant. According to the logic of cooperation in partnerships, this is about the worst way to organize 
a partnership. Under a sharing rule with a residual claimant, the other partners have every incentive to shirk. None-the-less, these partnerships were extremely successful.

Things were even more puzzling in the Kosovo crisis. Initially, the Europeans wanted to solve this problem amongst themselves in a partnership of equals. The result was a lot of political wrangling, a form of shirking. When the USA came in and took the lead things improved rapidly. Although it acted as a political residual claimant, only after the USA got involved did the partnership start to function effectively.

We argue that these apparent paradoxes can be explained by the model introduced in this paper. In these examples the partners face ambiguity about the effort provided by the governments to endure the tensions caused by the confrontation with the Warsaw pact, Iraq and Serbia respectively. The political pay-offs of the partnerships were divided according to the entrepreneurial sharing rule. The USA had the position of the entrepreneur, almost being a residual claimant.

In the second war against Iraq the main difference was that the USA was eager from the start not to allow any ambiguity with respect its to intentions. In the absence of ambiguity about its efforts, the initial support in the Security Council soon evaporated.

Other examples can be found in the context of interbank lending. Here the partners are the commercial banks and the regulator, typically the central bank. The latter is the residual claimant vis-a-vis the former. As noted in Rochet and Tirole (1996), central banks are known to pursue a strategy of "constructive ambiguity" to discourage shirking by their commercial partners.

\section{The CEU Framework}

It has long and widely been recognized that a fundamental distinction should be made between two types of decision problems in the face of uncertainty. In the first the uncertainty is about events that are considered to be of a familiar type. The second type of uncertainty concerns situations in which the decision maker has no experiences of himself or others to rely on. Following the discussion of Knight (1921), the former situations are usually referred to as 
situations of risk, the latter as situations of ambiguity. ${ }^{16}$

We use the Schmeidler (1989)-framework to represent ambiguity, i.e., we assume agents maximize their expected payoff with respect to non-additive probabilities. Non-additive probabilities, also called capacities, represent the ambiguity an agent faces about the relative frequencies with which outcomes are likely to occur. To evaluate the expected utility of an agent, we take the Choquet integral of his von Neumann-Morgenstern utility index with respect to the capacity.

A capacity $\nu$ over $S$ is convex if for any events $A, B \subseteq S$ we have ${ }^{17}$

$$
\nu(A)+\nu(B) \leq \nu(A \cup B)+\nu(A \cap B) .
$$

A capacity is additive if for any $A, B$ in the above, equality holds. An additive capacity is a probability distribution over $S$.

The concept of the ambiguity level of an event is introduced in Dow and Werlang (1992). It measures the ambiguity as the amount of the total mass of 1 that is assigned neither to the event, nor to its complement. The ambiguity level of a capacity $\nu$ at an event $E \subseteq S$ is

$$
\Psi_{\nu}(E):=1-\nu(S \backslash E)-\nu(E) .
$$

For convex capacities, ambiguity levels attain non-negative values only. In the case of additivity the ambiguity level is 0 .

Given a (convex) capacity $\nu$ that describes the non-additive probabilities assigned to the different events $E \subseteq S$, we evaluate the expected value of a function by applying the Choquet integral. ${ }^{18}$ If a decision-maker's beliefs are represented by a convex capacity, then he puts more weight on bad outcomes than an expected utility maximiser would.

As an example consider a capacity $\nu$ and an ambiguous event $E$, i.e. $\Psi_{\nu}(E)>0$. Let $u(s)=u_{1}$ for states $s \in E$ and $u(s)=u_{2}$ for the remaining states $s \in S \backslash E$.

For $u_{1}>u_{2}$ the Choquet integral of $u$ with respect to $\nu$ equals

$$
\begin{aligned}
& u_{1} \cdot \nu(E)+u_{2} \cdot[1-\nu(E)] \\
= & u_{1} \cdot \nu(E)+u_{2} \cdot \nu(S \backslash E)+u_{2} \cdot \Psi_{\nu}(E) .
\end{aligned}
$$

\footnotetext{
${ }^{16}$ Sometimes referred to as Knightian Uncertainty.

${ }^{17}$ For the definition of a capacity see Appendix, Definition 1.

${ }^{18}$ For a definition of the Choquet Integral see Appendix, Definition 2.
} 
For $u_{1}<u_{2}$, we obtain

$$
u_{1} \cdot \nu(E)+u_{2} \cdot \nu(S \backslash E)+u_{1} \cdot \Psi_{\nu}(E) .
$$

In each case, the bad outcome is "over-weighted" by the ambiguity level $\Psi_{\nu}(E)$ of the ambiguous event $E$.

Due to the Choquet integral it is meaningful to speak of maximizing expected utility when beliefs are represented as capacities. The resulting theory of individual decision making is known as Choquet Expected Utility (henceforth CEU). We now turn to games in which players face ambiguity about the strategy choices of the other players.

A game can be interpreted as a set of interconnected optimization problems, in which the equilibrium concept ensures the consistency of the strategy choices and beliefs of the players. So, one may think, it should not pose too many problems to extend CEU-decision theory to games. Unfortunately, things are not this easy. In the context of individual decision making, the ambiguity is about the states of nature. In games, ambiguity may be about the strategy choices of the other players as well.

Natural generalizations of Nash equilibrium in mixed strategies to CEU-games use the concept of equilibrium in non-additive beliefs. ${ }^{19}$ Consider a normal form game, which consists of a set of players $I$. The basic idea is that an equilibrium in this game is a profile of strategies and (non-additive) beliefs $\left(s_{i}^{*}, \nu_{i}^{*}\right)_{i \in I}$, such that for each player $i:{ }^{20}$

- the equilibrium strategy $s_{i}^{*}$ is a best response given his belief $\nu_{i}^{*}$ about the strategy choices $s_{-i}:=\left(s_{1}, \ldots, s_{i-1}, s_{i+1}, \ldots, s_{n}\right)$ of the other players and

- the capacity $\nu_{i}^{*}$ represents a situation where player $i$ "believes" his opponents will use the profile of equilibrium strategies $s_{-i}^{*}$.

We interpret "believes" $s_{-i}^{*}$ as meaning $s_{-i}^{*}$ lies in the support of $\nu_{i}^{*} \cdot{ }^{21}$ For the special case of additive beliefs, the support should exclude the states that occur with probability zero.

\footnotetext{
${ }^{19}$ This concept is introduced in one form or another in, e.g., Dow and Werlang (1994), Marinacci (2000) and Eichberger and Kelsey (2000). Alternative notions of equilibrium under uncertainty have been developed by, e.g., Epstein (1997), Groes et.al. (1998), Klibanoff (1996) and Lo (1996).

${ }^{20}$ For a formal definition of an equilibrium in non-additive beliefs see Appendix, Definition 4.

${ }^{21}$ For a formal definition of support see Appendix, Definition 3 .
} 
This equilibrium concept generalizes Nash equilibrium in mixed strategies. ${ }^{22}$ Nash equilibrium in pure strategies is a restriction of Nash equilibrium in mixed strategies (or in an interpretation that is more appropriate in our context in mixed beliefs) to those equilibria in which only pure strategies (pure beliefs) are used. So we face the question what restriction on capacities is the proper CEU-counterpart of the restriction to pure strategies.

As the generalization of pure strategies to CEU-games we consider capacities that have a unique singleton support. If $S$ is finite, the support of $\nu$ equals the set of points that have positive mass. A capacity which has the event $\left\{s^{*}\right\}$ as its unique support is called a pure belief with centre $s^{*}$. Every event that contains the centre of the pure belief has a positive mass. Any event that does not contain this centre has mass zero. ${ }^{23}$

As pure beliefs are introduced to be the non-additive counterparts of pure strategies, an equilibrium in pure beliefs is obtained as an equilibrium in non-additive beliefs, where the non-additive beliefs are restricted to being pure. As a consequence, a combination of strategies and beliefs $\left(s_{i}^{*}, \nu_{i}^{*}\right)_{i \in I}$ is an equilibrium in pure beliefs if the belief of every player is pure and has the strategy choices of the other players as its centre. ${ }^{24} 25$

We interpret an equilibrium in pure beliefs as describing a situation in which player $i$ "believes" that any other player $j$ will play strategy $s_{j}^{*}$ but does not have full confidence in this belief. This lack of confidence is represented by the non-additivity of player $i$ 's belief. ${ }^{26}$

\section{The Entrepreneurial Sharing Rule}

4.1. The Partnerships Model. Our model has $n \geq 2$ agents, who cooperate in a partnership. Each agent $k=1, \ldots, n$ chooses an effort level $0 \leq s_{k} \leq 2$, where $s_{k}$ is a multiple of a given rational number $\Delta=\frac{1}{r}$ with $r \in \mathbb{N}{ }^{27}$

For any effort level $s_{k}$ his corresponding cost is $c_{k}\left(s_{k}\right)$. We assume $c_{k}$ is a strictly increasing,

\footnotetext{
${ }^{22}$ Apart from allowing for non-additive beliefs, this solution concept does not require a player to believe that his opponents act independently.

${ }^{23}$ For a formal definition of a pure belief, see Appendix, Definition 5.

${ }^{24}$ For a formal definition of equilibrium in pure beliefs, see Appendix, Definion 6.

${ }^{25}$ This concept of equilibrium is used in Eichberger and Kelsey (2002).

${ }^{26}$ As is well-known Nash equilibrium in pure strategies may fail to exist. This is also the case for Equilibrium in pure beliefs as is show in Appendix, Example 1.

${ }^{27}$ To ensure there is a nontrivial moral hazard problem even though we restrict attention to a finite number of effort levels, we assume that $\Delta$ is "sufficiently small" to allow for a "sufficiently close" approximation of the effort levels in the continuous case. See Appendix, Assumption 4.
} 
strictly convex and differentiable function with $c_{k}(0)=0$ and $c_{k}^{\prime}(1)=1$. This implies $c_{k}(1)<1$. For the analysis in this paper, we assume that each agent has the same cost of effort function $c$.

The production of the partnership for the effort levels $\left(s_{1}, \ldots, s_{n}\right)$ is given by their sum

$$
\varphi\left(s_{1}, \ldots, s_{n}\right):=\sum_{k=1}^{n} s_{k} .
$$

A sharing rule assigns each agent a possibly negative share of the production of the partnership, such that the budget balance condition holds. ${ }^{28}$ I.e. the total production $\varphi$ is always shared amongst the partners. We assume that there are no binding limited liability constraints on the partnership.

In the absence of ambiguity, the utility level obtained by agent $k$ for given effort levels $\left(s_{1}, \ldots, s_{n}\right)$ equals his share in the total production minus his effort costs. In the presence of ambiguity, his non-additive belief $\nu_{k}$ also plays a role in determining his utility. Instead of his share in the total output, he considers its Choquet expected value according to his belief. He now chooses his effort level to maximize the Choquet expected value of his share in the production minus his cost of effort. ${ }^{29}$

In the absence of ambiguity an outcome, i.e., a profile of effort levels, is efficient if there is no combination of effort levels and a lump-sum redistribution of output that increases the utility of one partner without decreasing the utility of any other. In the context of our model, such efficiency is obtained when the sum of the utilities is maximized. In the absence of ambiguity, the efficient effort level of each agent equals one.

Accordingly, an outcome is ex-ante (ex-post) efficient when it maximizes the sum of the ex-ante (ex-post) utilities. A failure to obtain ex-post efficiency indicates that ambiguity leads to equilibrium effort choices in the partnership that differ from the efficient ones in its absence. Ex-ante efficiency, in addition, takes the ex-ante utility losses due to ambiguity aversion into account. ${ }^{30}$

As a point of reference, one may wish to consider partnerships with a linear sharing rule,

\footnotetext{
${ }^{28}$ For a formal definition see Appendix, To Section 4.

${ }^{29}$ For a formal representation see Appendix, To Section 3.

${ }^{30}$ When comparing two sharing rules, dominance according to ex-ante efficiency does not imply dominance according to ex-post efficiency. Neither does the reverse hold.
} 
where the agents may have differing cost functions. For such partnerships, the presence of ambiguity does not change the equilibrium effort levels. The intuition is that for any given partner, even though the total benefit from the partnership is perceived as ambiguous, there is no ambiguity concerning either the marginal cost or the marginal benefit, the latter being constant due to the linearity of both the production function and the sharing rule. Hence, the optimal choice of effort is not affected by introducing ambiguity. ${ }^{31}$

4.2. Entrepreneurial sharing rules. We introduce a class of sharing rules which exploit strategic ambiguity to reduce the moral hazard problem. We propose to treat partner 1 (the entrepreneur) differently from the other partners $j=2, \ldots, n$ (the workers), whereas the workers are treated equally. The sharing rules $\left(f_{1},\left(f_{j}\right)_{j=2, \ldots, n}\right)$ depend on a fine $G \geq 0$ and take the following form. For total production $\varphi$ the pay-out to the entrepreneur equals:

$$
f_{1}(\varphi):=\left\{\begin{array}{cl}
\varphi-(n-1) & \text { if } \varphi \geq 1 \\
(n-1) \cdot G+\varphi & \text { if } \varphi \in[0,1)
\end{array}\right.
$$

and each worker obtains:

$$
f_{j}(\varphi):=\left\{\begin{array}{cl}
1 & \text { if } \varphi \geq 1 \\
-G & \text { if } \varphi \in[0,1) .
\end{array}\right.
$$

We refer to $\left(f_{1},\left(f_{j}\right)_{j=2, \ldots, n}\right)$ as an entrepreneurial sharing rule. Its interpretation is as follows. When the total output $\varphi$ is at least 1 , the entrepreneur receives the difference between the output and the wages of the workers. If the output exceeds $n-1$ he makes a profit. If it falls short of $n-1$ to any amount that does not imply that all workers must have shirked, then the entrepreneur alone is liable for the shortfall. Since he obtains the production, but must pay each worker one unit of output, he incurs a loss. If the production of the partnership indicates that every worker must have shirked, then each worker pays the fine $G$. The entrepreneur obtains both the output and the fines. ${ }^{32}$

In the absence of ambiguity, no equilibrium in pure strategies exists for entrepreneurial sharing rules. The only serious candidate for an equilibrium has a total supply of 1 unit of effort. If the entrepreneur provides zero effort, he can improve by providing 1 unit, to increase

\footnotetext{
${ }^{31}$ A similar result holds for sharing rules with a budget breaker. For a formal proof, we refer to Kelsey and Spanjers (1997, Prop. 12).

${ }^{32}$ In reality the fine may correspond to a penalty such as losing promotion prospects.
} 
the production from 1 to 2 . On the other hand, if he provides a positive amount of effort while the output equals 1 , he can improve by reducing his effort by the minimal amount $\Delta$. The output would drop from 1 to $1-\Delta$ and the entrepreneur would not have to pay the workers. Rather, he receives the fine $G$ from each of them, as well as the output $1-\Delta$. Therefore, no equilibrium with a production of 1 exists.

\section{An EXAmple}

5.1. The Partnership. The partners $A$ and $B$ cooperate in a partnership where each of them has the effort levels 0,1 and 2 to choose from. The production function is $\varphi\left(s_{A}, s_{B}\right):=$ $s_{A}+s_{B}$. For each agent the cost of effort is given by $c(s):=\frac{2}{3} s^{\frac{3}{2}}$. The highest surplus that can be implemented in the absence of ambiguity is $\frac{1}{3}$, whereas the efficient outcome is obtained at a surplus of $\frac{2}{3}$. The latter requires that both agents put in an effort of 1 . Side payments are allowed for and neither partner faces constraints caused by limited liability.

To implement the ex-post efficient effort levels, the following entrepreneurial sharing rule is agreed upon:

$$
\begin{aligned}
f_{A}(\varphi) & :=\left\{\begin{array}{cc}
\varphi-1 & \text { if } \varphi \geq 1 \\
2 & \text { if } \varphi=0
\end{array}\right. \\
f_{B}(\varphi) & :=\left\{\begin{array}{cc}
1 & \text { if } \varphi \geq 1 \\
-2 & \text { if } \varphi=0
\end{array}\right.
\end{aligned}
$$

Thus, partner $A$ is an entrepreneur and partner $B$ is the worker employed by him. As long as it cannot be proven that the worker shirked, he receives a wage of 1 and the entrepreneur bears the costs of any shortfall of the output target. If the output equals 0 , the worker must have shirked and he pays a fine of 2 .

5.2. The Worker. Consider the pay-offs of the worker. For given belief $\nu_{B}$, his utility for a given effort level $s_{B}$ is the difference between his Choquet expected share of total production and his cost of effort. When his belief $\nu_{B}$ is pure, he believes the entrepreneur will use a pure strategy and his Choquet expected utility equals

$$
\begin{aligned}
& \nu_{B}(\{1,2\}) \cdot 1+\left[1-\nu_{B}(\{1,2\})\right] \cdot(-2)-c(0)=3 \cdot \nu_{B}(\{1,2\})-2 \quad \text { if } s_{B}=0 \\
& \nu_{B}(\{0,1,2\}) \cdot 1-c\left(s_{B}\right) \quad=1-c\left(s_{B}\right) \quad \text { if } s_{B} \geq 1 \text {, }
\end{aligned}
$$


irrespective of which pure strategy the entrepreneur is believed to choose. The unique best response of the worker is $s_{B}^{*}=1$, whenever the latter expression exceeds the former, i.e. when

$$
1-c(1)>3 \cdot \nu_{B}(\{1,2\})-2
$$

Substituting $c(1)=\frac{2}{3}$ and rearranging yields

$$
\nu_{B}(\{1,2\})<\frac{7}{9}
$$

If the centre of the belief $\nu_{B}$ is 0 , the inequality holds since $\nu_{B}(\{1,2\})=0$. If it is 1 or 2 we have $\nu_{B}(\{0\})=0$ and the inequality is satisfied for $\Psi_{\nu_{B}}(\{0\})>\frac{2}{9}$.

Thus, the worker provides one unit of effort whenever he perceives sufficient ambiguity about possible shirking by the entrepreneur.

5.3. The Entrepreneur. Now we turn to the entrepreneur, partner $A$. When the worker supplies one unit of effort, the pure equilibrium belief $\nu_{A}^{*}$ has its centre at 1 . For such belief, the Choquet expected utility of the entrepreneur is

$$
U_{A}\left(s_{A}\right):=\left\{\begin{array}{cc}
0 & \text { if } s_{A}=0 \\
\nu_{A}(\{1,2\})-\frac{2}{3} & \text { if } s_{A}=1 \\
\leq 0 & \text { if } s_{A}=2
\end{array}\right.
$$

Therefore, his unique best response is $s_{A}^{*}=1$, whenever $\nu_{A}^{*}(\{1,2\})>\frac{2}{3}$. Since the centre of the belief is 1 , we have $\nu_{A}^{*}(\{1,2\})=1-\Psi_{\nu_{A}^{*}}(\{0\})$. Thus, $s_{A}^{*}=1$ is a best response whenever $\Psi_{\nu_{A}^{*}}(\{0\})<\frac{1}{3}$.

So the entrepreneur does not shirk when the worker provides one unit of effort and the entrepreneur's perceived ambiguity about possible shirking by the worker is sufficiently low.

5.4. Equilibrium. The corresponding equilibrium in the partnership is the profile $\left(s_{k}^{*}\right.$, $\left.\nu_{k}^{*}\right)_{k \in\{A, B\}}$ such that for each partner $k \in\{A, B\}$ we have $s_{k}^{*}=1$ and

$$
\nu_{k}^{*}(E):=\left\{\begin{array}{cl}
0 & \text { if } 1 \notin E \\
\in\left(0,1-\Psi_{\nu_{k}}(\{0\})\right] & \text { if } E=\{1\} \\
1-\Psi_{\nu_{k}}(\{0\}) & \text { if } E=\{1,2\} \\
1 & \text { if } E=S
\end{array}\right.
$$

where both $\nu_{A}^{*}(\{0,1\})$ and $\nu_{B}^{*}(\{0,1\})$ can take arbitrary values, provided the beliefs remain convex capacities and both $\Psi_{\nu_{A}^{*}}(\{0\})<\frac{1}{3}$ and $\Psi_{\nu_{B}^{*}}(\{0\})>\frac{2}{9}$ hold. 
Accordingly, the entrepreneur faces sufficiently little ambiguity with respect to the possible shirking of the worker, whereas the worker perceives sufficient ambiguity with respect to the effort provided by the entrepreneur.

5.5. Ex-post Efficiency. In this partnership, there exists an ex-post efficient equilibrium with symmetric beliefs whenever $\Psi_{\nu_{A}^{*}}(\{0\})=\Psi_{\nu_{B}^{*}}(\{0\}) \in\left(\frac{2}{9}, \frac{1}{3}\right)$. Intuitively, symmetric beliefs represent a situation where $A$ and $B$ both perceive the same "amount" of ambiguity. The corresponding total production is $\varphi\left(s_{A}^{*}, s_{B}^{*}\right)=2$ and the ex-ante equilibrium utility levels are $U_{A}=\frac{1}{3}-\Psi_{\nu_{A}^{*}}(\{0\})$ and $U_{B}=\frac{1}{3}$.

5.6. Ex-ante Efficiency. In order to obtain ex-ante efficiency, we must have $U_{A}=U_{B}=$ $\frac{1}{3}$. This holds when $\Psi_{\nu_{A}^{*}}(\{0\})=0$. The entrepreneur may still face ambiguity with respect to the effort provided by the worker, but the possibility that the worker shirks no longer enters his calculations. Amongst others, this condition is satisfied if the belief of the entrepreneur is additive.

\section{EqUiLIBRIUM AND EFFICIENCY}

The results of the example also apply to our more general model of partnerships with entrepreneurial sharing rules. The first result we state is that an equilibrium in pure beliefs in the partnership exists. If the workers perceive sufficient ambiguity, then their best response is to choose the effort level of one, irrespective of the effort provided by the other workers or the entrepreneur. Whatever his belief, some action of the entrepreneur is a best response. The resulting profile of effort levels and beliefs constitutes an equilibrium in pure beliefs.

Theorem 1. ${ }^{33}$ In the game induced by the entrepreneurial sharing rule, there exists an equilibrium in pure beliefs where

(i) the ambiguity the entrepreneur faces, that the workers provided at least their planned effort, is sufficiently small and

(ii) the ambiguity of each worker, regarding the event that the other partners (including the entrepreneur) provide any effort at all, is sufficiently large.

\footnotetext{
${ }^{33}$ For a formal and slightly more general representation of the Theorem see Appendix, Theorem 5 .
} 
In every equilibrium in pure beliefs with (i) and (ii), all partners provide the efficient levels of effort. ${ }^{34}$

For any given non-negative fine and suitable restrictions on the beliefs of the agents, the equilibrium actions are unique and equal to the efficient effort levels. Under these restrictions, every equilibrium is ex-post efficient. ${ }^{35}$

Theorem 1 indicates that the ex-post efficient outcome can be implemented by the partnership, but what about ex-ante efficiency? The above result fails to take into account the ex-ante utility loss due to ambiguity aversion. In equilibrium, the workers perceive ambiguity about the effort levels of the other agents, but they do not face ambiguity with respect to the payment they obtain. By choosing an effort level of one, any worker ensures that, independent of the effort provided by others, he obtains his wage of one. Thus, in equilibrium no worker perceives ambiguity about his payoff and no ex-ante utility is lost because of it.

So the only agent who may face such a utility loss is the entrepreneur. If his belief does not rule out the workers providing a total effort less than $n-1$, he experiences an ex-ante loss of utility. If he rules this possibility out, then his Choquet expected utility is not affected by the ambiguity. Ambiguity is present only out of equilibrium, where it prevents defections. But in equilibrium it does not lead to ex-ante utility losses. Hence, ex-ante efficiency is obtained. As a special case, we find that ex-ante efficiency is achieved when the pure belief of the entrepreneur is additive.

In some respects, the role of ambiguity is similar to that of risk in Rasmusen (1987). He proposes to conduct a lottery in the case some of the partners shirked. When the partners are risk-averse, this leads to an ex-post loss of utility and may therefore alleviate the moral hazard problem. Andolfatto and Nosal (1997) argue that Rasmusen's solution is not credible. After shirking occurred, the team members will renegotiate the contract to prevent the lottery being held. A similar argument cannot be made in the context of our model as it contains no equivalent to Rasmusen's lottery. When an agent shirks, the strategic ambiguity leads to a

\footnotetext{
${ }^{34}$ It should be noted that the efficiency result crucially depends on each worker having the same efficient effort level. Thus, considering partnerships with identical agents simplifies our analysis and limits our results.

${ }^{35}$ In the absence of side payments and confidence enhancing measures, the equilibrium is also coalition proof. This is shown in Kelsey and Spanjers (1997).
} 
loss in utility before the production of the partnership is known. Therefore, this loss cannot be renegotiated away afterwards.

Theorem 2. ${ }^{36}$ If the entrepreneur perceives no ambiguity that the workers provided at least their planned effort and the belief of each worker contains sufficient ambiguity that the other partners (including the entrepreneur) do not provided any effort, then every equilibrium is ex-ante efficient.

Theorem 2 states that the ex-ante efficient outcome of the partnership is implemented by an entrepreneurial sharing rule. The only remaining problem is that the beliefs of the agents fail to be identical. As indicated in, e.g., Kelsey and Nandeibam (1996), one may interpret the capacities that represent the beliefs of the agents as describing their ambiguity aversion. Under this interpretation, the aversion to ambiguity is a characteristic that should be part of the description of the agents, rather than an equilibrium property. By allowing agents to have different equilibrium beliefs, one may argue, the assumption of identical agents is implicitly abandoned.

The following corollary states that ex-post efficiency can be obtained as an equilibrium in identical pure beliefs. Thus, any asymmetry stems from the sharing rule, i.e., from the contract that constitutes the partnership.

Corollary 3. Consider a partnership with an entrepreneurial sharing rule. Let the fine be sufficiently large. Then there exists an ex-post efficient equilibrium in pure beliefs, in which the agents have identical beliefs about the aggregate behaviour of the others.

\section{CONCluding Remarks}

7.1. Rationality. The results we obtained rest on how ambiguity affects the behaviour of the agents. In our example, the worker provides the efficient amount of effort to insure himself against potential shirking by the entrepreneur. But in equilibrium the entrepreneur does not shirk. So the question may arise whether the results depend on a belief that is systematically mistaken.

\footnotetext{
${ }^{36}$ For a formal and slightly more general statement of the Theorem see Appendix, Theorem 6 .
} 
In our interpretation, this is not the case. Rather, the worker correctly believes the entrepreneur does not shirk, but has only limited confidence in this belief. As a result he acts cautiously and puts in effort to avoid the fear of facing the penalty for shirking. Unlike the expected utility model, our model of ambiguity is capable of distinguishing different degrees of confidence in a belief. In an equilibrium in pure beliefs the worker assumes the entrepreneur does not shirk. But for less than full confidence, the fear that the entrepreneur may neverthe-less shirk affects the worker's utility level.

Another question that may arise is whether a worker with beliefs as in our model can still be considered to be rational. The answer to this question depends upon which definition of rationality one adopts. The agents in our model are maximizing a complete and transitive preference, which is the standard definition of rationality. Given individuals have a dislike of ambiguity, it is surely rational to act in a manner which reduces ones exposure to it.

In the context of choice under uncertainty, some economists would adopt the stronger definition of rationality that only expected utility preferences are rational. It could be argued that our model even satisfies this stronger definition of rationality if it is a reduced form of a more complex model, in which individuals are acting to protect themselves against possible mis-specifications of the model. The point is argued in more detail in Kelsey and Milne (1999) and Mukerji (1997). Intuitively, an argument for ambiguity is that it applies in situations in which individuals do not completely understand the environment in which they are operating.

Our preferred interpretation is that ambiguity aversion implies a small amount of bounded rationality. In our view, this is well justified both by intuition and by experimental evidence of ambiguity aversion.

7.2. Dynamics. In an intertemporal extension of our model would beliefs tend to be contradicted over time? Our key result is that cooperation is sustained by the threat of an ambiguous punishment off the equilibrium path. Since punishment never happens in equilibrium, there is no scope to update beliefs. The ambiguous threat is never tested, hence ambiguity does not necessarily dissipate over time. Provided the worker puts in the efficient amount of effort, he gets his wages. He will never learn whether or not the entrepreneur shirked. 
So what happens if we are repeatedly in the same situation and the worker can learn whether or not the entrepreneur shirked? A complete answer is not possible as this is an area of current research. There has been some theoretical work on updating non-additive beliefs. Consider a decision maker who is repeatedly faced with ambiguous urns. It has been shown that if the decision-maker repeatedly faces with the same ambiguous urn, then eventually his beliefs will converge to an additive probability distribution. ${ }^{37}$ In other words, ambiguity will decrease over time.

If, however, an individual is faced with repeated choices from different ambiguous urns, then ambiguity will persist over time. ${ }^{38}$ We believe that many actual economic decisions are more like sampling from different ambiguous urns. Therefore we believe that ambiguous threats could also be used to sustain cooperation in a consistent intertemporal model.

7.3. Optimism. One may argue that contrary to our assumption of pessimism, in practice people are excessively optimistic. These two observations are not contradictory since it is possible for both good and bad outcomes to be over-weighted at the expense of intermediate outcomes. This would induce behaviour which is at the same time optimistic and pessimistic. Consider the following form of preferences

$$
V(a)=\gamma \cdot \mathbb{E}\{u(a(s))\}+\beta \cdot(1-\gamma) \cdot M(a)+(1-\beta) \cdot(1-\gamma) \cdot m(a)
$$

where $M(a):=\max _{s \in S}\{u(a(s))\}$ denotes the maximum utility and $m(a):=\min _{s \in S}\{u(a(s))\}$ the minimum utility associated with the act $a$. Furthermore, $\beta$ reflects the level of optimism/pessimism, whereas $\gamma$ represents the level of confidence in the probability assessment. ${ }^{39}$ These preferences put more weight on both good and bad outcomes than an expected utility maximiser would. Thus they may be described as displaying optimism and pessimism at the same time.

People do certainly behave optimistically at times buying lottery tickets, entering risky careers such as acting and, until recently, buying shares of new economy stocks. The degree of optimism/pessimism depends both on the subject and on the context.

\footnotetext{
${ }^{37}$ See Marinacci (2002).

${ }^{38}$ See Marinacci (1999).

${ }^{39}$ Similar preferences have been axiomatised by Chateauneuf, Eichberger and Grant (2002).
} 
In this paper we abstracted from optimism and focused on the implications of pessimism for sustaining cooperation. The possibility of optimism reinforces our argument, since it would allow cooperation to be sustained by ambiguous promises as well as ambiguous threats. This would further expand the range of outcomes which can be implemented.

Despite this, in one sense, our paper does model an optimistic attitude of the entrepreneur. the incentive scheme in our paper relies on the worker having a high degree of ambiguityaversion (or pessimism) and the entrepreneur having a low degree of ambiguity aversion. Thus in a relative sense the entrepreneur is more optimistic. We believe this is compatible with Knight's arguments that more optimistic (i.e., less pessimistic) individuals are more willing to face ambiguity and tend to become entrepreneurs.

\section{REFERENCES}

Andolfatto, D. And E. Nosal (1997), “Optimal Team Contracts”, Canadian Journal of Economics, Vol. 30, pp. 385-396.

Bernardo, A. AND I. Welch (2001), "On the Evolution of Overconfidence and Entrepreneurs", Yale Cowles Foundation, Discussion Paper No. $130 \%$.

Bolton, G. AND R. Zwick (1995), “Anonymity versus Punishment in Ultimatum Bargaining", Games and Economic Behaviour, Vol. 10, pp. 95-121.

Camerer, C. And M. Weber (1992), "Recent Developments in Modelling Preferences: Uncertainty and Ambiguity", Journal of Risk and Uncertainty, Vol. 5, pp. 325-370.

Chateauneuf, A. J. Eichberger and S. Grant (2002), "Choice under Uncertainty with the Best and the Worst in Mind: NEO-Additive Capacities", Working Paper, Heidelberg University.

Camerer, C. (1997), "Progress in Behavioral Game Theory", Journal of Economic Perspectives, Vol. 11, pp. 167-188.

Davis, D. And C. Holt (1993), Experimental Economics, Princeton University Press, New Jersey. 
Dow, J. And S. Werlang (1992), "Risk Aversion, Uncertainty Aversion and the Optimal Choice of Portfolio", Econometrica, Vol. 60, pp. 197-204.

Dow, J. AND S. Werlang (1994), "Nash Equilibrium under Knightian Uncertainty: Breaking Down Backward Induction", Journal of Economic Theory, Vol. 64, pp. 305-324.

Eichberger, J. And D. Kelsey (2000), "Non-Additive Beliefs and Strategic Equilibria", Games and Economic Behavior, Vol. 30, pp. 183-215.

Eichberger, J. AND D. Kelsey (2002), "Strategic Complements, Substitutes and Ambiguity: The Implications for Public Goods", Journal of Economic Theory, Vol. 106, pp. 436-466. Ellsberg, D. (1961), "Risk, Ambiguity and the Savage Axioms", Quarterly Journal of Economics, Vol. 75, pp. 643-669.

Epstein, L. (1997), "Preference, Rationalizability and Equilibrium", Journal of Economic Theory, Vol. 73, pp. 1-29.

Eswaran, M. And A. Kotwal (1984), "The Moral Hazard of Budget-Breaking”, Rand Journal of Economics, Vol. 15, pp. 578-581.

Forsythe, R., J. Horowitz, E. SAvin And M. Sefton (1994), "Fairness in Simple Bargaining Experiments", Games and Economic Behaviour, Vol. 6, pp. 347-369.

Gervais, S. J. Heaton and T. Odean (2000), "Capital Budgeting in the Presence of Managerial Overconfidence and Optimism", Mimeo, Finance Department, Wharton School, University of Pennsylvania.

GilboA, I. (1987), "Expected Utility with Purely Subjective Non-Additive Probabilities", Journal of Mathematical Economics, Vol. 16, pp. 65-88.

Gonzalez, R. And G. Wu (1999), "On the Shape of the Probability Weighting Function", Cognitive Psychology, Vol. 38, pp. 129-166.

Groes, E., Jacobsen, H.J., Sloth, B. and T. Tranaes (1998), "Nash Equilibrium in Lower Probabilities", Theory and Decision, Vol. 44, pp. 37-66.

HALler, H. (1985), "Separation of Ownership and Labour: Welfare Considerations", Zeitschrift für Nationalökonomie (Journal of Economics), Vol. 45, pp. 245-265. 
Haller, H. (2000), "Non-Additive Beliefs in Solvable Games", Theory and Decision, Vol. 49, pp. 313-338.

Hofman, E. K. McCabe, K. Shacat And V. Smith (1994), "Preferences, Property Rights and Anonymity in Bargaining Games", Games and Economic Behaviour, Vol. 7, pp. 346-380. Holmström, B. (1982), "Moral Hazard in Teams", Bell Journal of Economics, Vol. 13, pp. 324-340.

Kelsey, D. And F. Milne (1999), "Induced Preferences, Nonadditive Beliefs, and Multiple Priors", International Economic Review, Vol. 40, pp. 455-477.

Kelsey, D. And S. NAndeibam (1996), "On the Measurement of Uncertainty Aversion", Mimeo, University of Birmingham, Birmingham.

Kelsey, D. And W. Spanjers (1997), "Uncertainty in Partnerships", Department of Economics DP 97-16, University of Birmingham, Birmingham.

KiLKa, M. AND M. Weber (1998), "What Determines the Shape of the Probability Weighting Function under Uncertainty?", Management Science, Vol. 47, pp. 1712-1726.

Klibanoff, P. (1996), "Uncertainty, Decision, and Normal Form Games", Mimeo, Northwestern University, Evanston.

Knight, F. (1921), Risk, Uncertainty and Profit, Houghton Mifflin, Boston.

Legros, P. And H. Matsushima (1991), "Efficiency in Partnerships", Journal of Economic Theory, Vol. 55, pp. 296-322.

Lo, K. (1996), "Equilibrium in Beliefs under Uncertainty", Journal of Economic Theory, Vol. 71, pp. $443-484$.

MarinacCI, M. (1999), "Limit Laws for Non-Additive Probabilities and their Frequentist Interpretation", Journal of Economic Theory, Vol. 84, pp. 145-196.

MarinacCI, M. (2000), "Ambiguous Games", Games and Economic Behavior, Vol. 31, pp. $191-219$.

MarinacCI, M. (2002), "Learning from Ambiguous Urns", Statistical Papers, Vol. 43, pp. $143-151$. 
MukerJi, S. AND H. Shin (2002), "Is Common Knowledge Possible in Games with Knightian Uncertainty?", Advances in Theoretical Economics, 2.

MukerJi, S. (1997), "Understanding the Non-Additive Probability Decision Model", Economic Theory, Vol. 9, pp. 23-46.

Nandeibam, S. (1997), "Efficiency in Teams with Sub-Teams", Mimeo, The University of Birmingham, Birmingham.

Rasmusen, E. (1987), "Moral Hazard in Risk-Averse Teams", Rand Journal of Economics, Vol. 18, pp. 428-435.

Rigotti, L. And M. Ryan (1997), "Knightian Uncertainty, Entrepreneurship and Corporate Culture", Mimeo, University of Auckland, Auckland.

Rochet, J. And J. Tirole (1996), "Interbank Lending and Systematic Risk", Journal of Money, Credit and Banking, Vol. 28, pp. 733-765.

Roth, A. (1995), "Bargaining Experiments", in Handbook of Experimental Economics, ed. by J. Kagel and A. Roth, Princeton University Press, New Jersey.

SchmeIdleR, D. (1989), "Subjective Probability and Expected Utility without Additivity", Econometrica, Vol. 57, pp. 571-587.

\section{APPENDIX}

This appendix contains formal statements of our definitions and results. It concludes with the proofs of the theorems and the corollary.

\section{TO SECTION 3}

Definition 1. Let the set of states of nature be $S$, which we assume to be finite. Denote the set of events (i.e., subsets of $S$ ) by $P(S):=\{E \subseteq S\}$. A function $\nu: P(S) \rightarrow[0,1]$ is a capacity if it satisfies $\nu(\emptyset)=0, \nu(S)=1$, and for any $A, B \subseteq S$ with $A \subseteq B$ we have $\nu(A) \leq \nu(B)$. 
Definition 2. Let $\nu$ be a capacity and let $u: S \rightarrow \mathbf{R}$ be a function. Let $\pi:=\left(\pi_{1}, \ldots, \pi_{|S|}\right)$ be a permutation of the states of nature such that $u\left(\pi_{1}\right) \geq u\left(\pi_{2}\right) \geq \ldots \geq u\left(\pi_{|S|}\right)$. The Choquet integral of $u$ with respect to $\nu$ is

$$
\int^{C} u d \nu:=u\left(\pi_{1}\right) \cdot \nu\left(\left\{\pi_{1}\right\}\right)+\sum_{k=2}^{|S|} u\left(\pi_{k}\right) \cdot\left[\nu\left(\left\{\pi_{1}, \ldots, \pi_{k}\right\}\right)-\nu\left(\left\{\pi_{1}, \ldots, \pi_{k-1}\right\}\right)\right] .
$$

Definition 3. An event $E \subseteq S$ is a support of $\nu$ if $\nu(S \backslash E)=0$ and $F \subset E$ implies $\nu(S \backslash F)>0 .{ }^{40} 41$

Definition 4. Let for each player $i \in I$ the capacity $\nu_{i}^{*}: P\left(S_{-i}\right) \rightarrow[0,1]$ and the strategy $s_{i}^{*} \in S_{i}$ be given, where $S_{-i}:=\prod_{j \in I \backslash\{i\}} S_{j}$. The profile $\left(s_{i}^{*}, \nu_{i}^{*}\right)_{i \in I}$ is an equilibrium in non-additive beliefs if for each player $i$ there exists an event $E_{i}^{*}$ that is a support of $\nu_{i}^{*}$ such that

$$
s_{-i}^{*} \in E_{i}^{*} \subseteq \prod_{j \in I \backslash\{i\}} R_{j}\left(\nu_{j}^{*}\right)
$$

where

$$
R_{j}\left(\nu_{j}^{*}\right)=\arg \max _{s_{j} \in S_{j}} \int^{C} u_{j}\left(s_{j}, \cdot\right) d \nu_{j}^{*}
$$

is the set of best responses of player $j$ for given belief $\nu_{j}^{*}$.

Definition 5. A capacity $\nu: P(S) \rightarrow[0,1]$ is a pure belief with centre $s^{*} \in S$ if $\left\{s^{*}\right\}$ is the unique support of $\nu$. If $S$ is finite, the support of $\nu$ equals the set of points that have positive mass.

Definition 6. Let $\left(s_{i}^{*}, \nu_{i}^{*}\right)_{i \in I}$ be an equilibrium such that for each player $i \in I$ the capacity $\nu_{i}^{*}$ is a pure belief with centre $s_{-i}^{*}$. Then $\left(s_{i}^{*}, \nu_{i}^{*}\right)_{i \in I}$ is an equilibrium in pure beliefs.

Example 1. In general, an equilibrium in pure beliefs may fail to exist, as in the "matching pennies" game:

\begin{tabular}{|c|c|c|}
\cline { 2 - 3 } \multicolumn{1}{c|}{} & $L$ & $R$ \\
\hline$U$ & $(1,0)$ & $(0,1)$ \\
\hline$D$ & $(0,1)$ & $(1,0)$ \\
\hline
\end{tabular}

\footnotetext{
${ }^{40}$ There are several competing notions of a support of a capacity. The way the support of a capacity is defined may influence the set of equilibria of the game. For a discussion we refer to Haller (2000).

${ }^{41}$ It should be noted that the support of a capacity may fail to be unique. For examples see Dow and Werlang (1994).
} 
For contradiction, suppose that player 1 plays $U$ in an equilibrium in pure beliefs. Then the equilibrium belief of player 2 has its centre at $U$. It follows that $\nu_{2}(\{U\})>0$ and $\nu_{2}(\{D\})=0$. The Choquet expected payoff of player 2 when he chooses $L$ is $\nu_{2}(\{D\})+0 \cdot\left[1-\nu_{2}(\{D\})\right]=0$. For $R$ it equals $\nu_{2}(\{U\})+0 \cdot\left[1-\nu_{2}(\{U\})\right]>0$, and so the unique best response of player 2 is $R$. This implies that, in this equilibrium, the belief of player 1 is pure with centre $R$. For such belief his unique best response is $D$ and player 1 fails to play $U$. By a similar argument it follows that in equilibrium player 1 does not play $D$ either and therefore no equilibrium in pure beliefs exists.

\section{TO SECTION 4}

Let $r$ be a positive integer and denote $\Delta:=\frac{1}{r}$. Let $[\underline{x}, \bar{x}]_{\Delta}$ denote the set of all rational numbers between $\underline{x}$ and $\bar{x}$, which are integer multiples of $\Delta$. We denote $\left.(\underline{x}, \bar{x})_{\Delta}, \underline{[x}, \bar{x}\right)_{\Delta}$ and $(\underline{x}, \bar{x}]_{\Delta}$ accordingly.

Let each agent $k \in N:=\{1, \ldots, n\}$ choose an effort level from the set $S_{k}:=[0,2]_{\Delta}$. The production of the partnership for the effort levels $s:=\left(s_{1}, \ldots, s_{n}\right) \in S:=\prod_{k \in N} S_{k}$ is given by

$$
\varphi(s):=\sum_{k \in N} s_{k}
$$

A sharing rule is a profile $\left(g_{k}\right)_{k \in N}$ of functions that assign each agent a (possibly negative) share of the production of the partnership, such that the budget balance condition holds, i.e., for each $\varphi \in[0,2 n]_{\Delta}$ we have $\sum_{k \in N} g_{k}(\varphi)=\varphi$.

In the absence of ambiguity, the utility level obtained by agent $k$ for a given profile of effort levels $s:=\left(s_{1}, \ldots, s_{n}\right)$ equals $g_{k}(\varphi(s))-c\left(s_{k}\right)$. When he has a non-additive belief $\nu_{k}$, his decision problem becomes:

$$
\max _{s_{k} \in S_{k}} \int^{C} g_{k}\left(\varphi\left(s_{k}, s_{-k}\right)\right) d \nu_{k}-c\left(s_{k}\right) .
$$

We denote $[t, \rightarrow)_{\Delta}:=[t, 2(n-1)]_{\Delta}$. For notational convenience, we introduce the capacity $\widetilde{\nu}_{k}: P\left([0, \rightarrow)_{\Delta}\right) \rightarrow[0,1]$, which denotes the mass $\nu_{k}$ assigns to the event that the total effort 
of the agents other than $k$ is in some set $E$. So for each $E \subseteq[0, \rightarrow)_{\Delta}$ we have

$$
\widetilde{\nu}_{k}(E):=\nu_{k}\left(\left\{s_{-k} \in S_{-k} \mid \sum_{j \in N \backslash\{k\}} s_{j} \in E\right\}\right) .
$$

Assumption 4. For $c$ and $\Delta$ we have that

$$
\frac{\Delta}{n}<c(1)-c(1-\Delta)
$$

Assumption 4 states that $\Delta$ is "sufficiently small" to allow for a "sufficiently close" approximation of the effort levels in the continuous case. ${ }^{42}$

\section{TO SECTION 6}

For proofs, see the subsequent section of this appendix.

Theorem 5. ${ }^{43}$ In the game induced by the entrepreneurial sharing rule, there exists an equilibrium in pure beliefs $\left(\left(s_{k}^{*}\right)_{k \in N},\left(\nu_{k}^{*}\right)_{k \in N}\right)$, with

(i) $\Psi_{\widetilde{\nu}_{1}^{*}}\left(\left[\widetilde{s}_{-1}, \rightarrow\right)_{\Delta}\right)<1-\frac{1}{\Delta} \cdot[c(1)-c(1-\Delta)]$, where $\widetilde{s}_{-1}:=\sum_{j=2}^{n} s_{j}$ and

(ii) $\Psi_{\nu_{j}^{*}}\left(\left\{\underline{0}_{j}\right\}\right)>\frac{c(1)}{G+1}$ for each $j \in J$, where $\underline{0}_{-j}$ denotes each partner other than $j$ choosing effort level 0.

Moreover, in every equilibrium in pure beliefs with (i) and (ii), all partners provide the efficient levels of effort.

Theorem 6. Let the belief $\nu_{1}$ of agent 1 be pure such that if $s_{-1} \in S_{-1}$ is the centre of $\nu_{1}$ then $\Psi_{\widetilde{\nu}_{1}}\left(\left[\widetilde{s}_{-1}, \rightarrow\right)_{\Delta}\right)=0$. Let the belief $\nu_{j}$ of each $j \in J$ be restricted to be pure with $\Psi_{\nu_{j}}\left(\left\{\underline{0}_{-j}\right\}\right)>\frac{c(1)}{G+1}$. Then every equilibrium is ex-ante efficient.

\section{PROOFS TO SECTION 6}

\footnotetext{
${ }^{42}$ In particular, this assumption is satisfied whenever $c^{\prime}\left(1-\frac{1}{\Delta}\right) \geq \frac{\Delta}{n}$.

${ }^{43}$ In a stronger but less accessible formulation, the theorem generalizes to continuous strategy spaces by letting $\Delta \rightarrow 0$, without imposing $\Psi_{\widetilde{\nu}_{1}^{*}}\left(\left[\widetilde{s}_{1}, \rightarrow\right)\right)=0$.
} 
Lemma 7. Let $j \in J$ and let $\nu_{j}$ represent the pure belief of agent $j$. Let $\nu_{j}$ be such that for the ambiguity level of $\left\{\underline{\underline{Q}}_{j}\right\}$ we have $\Psi_{\nu_{j}}\left(\left\{\underline{\underline{L}}_{-j}\right\}\right)>\frac{c(1)}{G+1}$. Then the (unique) best response of agent $j$ is $s_{j}^{*}=1$.

\section{Proof of Lemma 7}

(i) $s_{j}=1$.

For $s_{j}=1$, we have for the Choquet expected utility of agent $j$ that

$$
\int^{C} f_{j}\left(\varphi\left(\underline{1}_{j}, s_{j}\right)\right) d \nu_{j}-c\left(s_{j}\right)=1-c(1)
$$

where $\underline{1}_{-j}$ denotes each partner other than $j$ choosing effort level 1 .

(ii) $s_{j}<1$.

For given $s_{j}<1$, and $G \geq 0$ we can make the Choquet expected utility of agent $j$

$$
\int^{C} f_{j}\left(\varphi\left(1_{-j}, s_{j}\right)\right) d \nu_{j}-c\left(s_{j}\right)
$$

smaller than $1-c(1)$ by choosing $\left.\Psi_{\nu_{j}}\left(\underline{0}_{-j}\right\}\right)$ sufficiently large (but, of course, less than 1 ). To see this, note that $\Psi_{\nu_{j}}\left(\left\{\underline{0}_{-j}\right\}\right)=1-\nu_{j}\left(\left\{\underline{0}_{-j}\right\}\right)-\nu_{j}\left(S_{-j} \backslash\left\{\underline{0}_{-j}\right\}\right)$. By definition, $\nu_{-j}$ is a pure belief, so if its centre is $s_{-j} \neq \underline{0}_{-j}$, we have $\nu_{j}\left(\left\{\underline{0}_{-j}\right\}\right)=0$ and $\nu_{j}\left(S_{-j} \backslash\left\{\underline{0}_{-j}\right\}\right)=1-\Psi_{\nu_{j}}\left(\left\{\underline{0}_{-j}\right\}\right)$. Note that:

$$
\left.\int^{C} f_{j}\left(\varphi\left(\underline{1}_{-j}, s_{j}\right)\right) d \nu_{j}-c\left(s_{j}\right) \leq\left[1-\Psi_{\nu_{j}}\left(\underline{0}_{-j}\right\}\right)\right] \cdot 1+\left[\Psi_{\nu_{j}}\left(\left\{\underline{0}_{-j}\right\}\right)\right] \cdot(-G)-0 .
$$

By choosing $\left.\Psi_{\nu_{j}}\left(\underline{0}_{j}\right\}\right)>\frac{c(1)}{G+1}$, the expected utility of agent $j$ becomes less than $1-c(1)$. If $\nu_{j}$ is pure with centre $s_{-j}=\underline{0}_{j}$, then $\nu_{j}\left(S_{-j} \backslash\left\{\underline{0}_{j}\right\}\right)=0$. It follows that the weight of the outcome $-G$ in the Choquet integral is $\Psi_{\nu_{j}}\left(\left\{\underline{\underline{L}}_{j}\right\}\right)=1-\nu_{j}\left(S_{-j} \backslash\left\{\underline{\underline{-}}_{-j}\right\}\right)=1$, and the Choquet expected utility of agent $j$ is less than $1-c(1)$.

(iii) $s_{j}>1$.

The Choquet expected utility of agent $j$ for $s_{j}=1+t \cdot \Delta>1$ for any integer $t>0$ equals, by a similar argument,

$$
\int^{C} f_{j}\left(\varphi\left(\underline{1}_{-j}, 1+t \cdot \Delta\right)\right) d \nu_{j}-c(1+t \cdot \Delta)=1-c(1+t \cdot \Delta)<1-c(1) .
$$


Therefore, $s_{j}=1$ is the unique best response of agent $j$.

Lemma 8. Let the capacity $\nu_{1}$ represent the belief of agent 1 . Let $\nu_{1}$ be pure with centre $\underline{1}_{-1}$, where $\underline{1}_{-1}$ denotes each partner other than 1 choosing effort level 1 . Let the ambiguity level $\Psi_{\widetilde{\nu}_{1}}\left([n-1, \rightarrow)_{\Delta}\right)<1-\frac{1}{\Delta} \cdot(c(1)-c(1-\Delta))$. Then the (unique) best response of agent 1 is $s_{1}^{*}=1$.

\section{Proof of Lemma 8}

Since $\nu_{1}$ is a pure belief at $\underline{1}_{-1}$ by assumption, we have that for each $A \in P\left(S_{-1}\right)$ with $\underline{1}_{-1} \notin A$ that $\nu_{1}(A)=0$. In particular, $\nu_{1}\left(S_{-1} \backslash\left\{\underline{1}_{-1}\right\}\right)=0$.

Since $\Psi_{\widetilde{\nu}_{1}}\left([n-1, \rightarrow)_{\Delta}\right):=1-\widetilde{\nu}_{1}\left([n-1, \rightarrow)_{\Delta}\right)-\widetilde{\nu}_{1}\left([0, n-1)_{\Delta}\right)$ is assumed to be less than $1-\frac{1}{\Delta} \cdot(c(1)-c(1-\Delta))$, we have $\widetilde{\nu}_{1}\left([n-1, \rightarrow)_{\Delta}\right)>\frac{1}{\Delta} \cdot(c(1)-c(1-\Delta))$.

(i) $s_{1}<1$.

The difference in the Choquet expected utility of agent 1 for choosing the effort level of 1 instead of $s_{1}<1$ is at least

$$
\begin{aligned}
\widetilde{\nu}_{1}\left([n-1, \rightarrow)_{\Delta}\right)-c(1)-\widetilde{\nu}_{1}\left([n-1, \rightarrow)_{\Delta}\right) \cdot s_{1}+c\left(s_{1}\right) \\
\quad>\frac{1}{\Delta} \cdot\left(c(1)-c\left(s_{1}\right)\right) \cdot\left(1-s_{1}\right)-c(1)+c\left(s_{1}\right) \\
\quad \geq \frac{1}{\Delta} \cdot(c(1)-c(1-\Delta)) \cdot(1-(1-\Delta))-c(1)+c(1-\Delta)=0
\end{aligned}
$$

by using $c^{\prime}(1)=1$, the strict convexity of $c$ and the structure of the sharing rule. So $s_{1}<1$ is not a best response.

(ii) $s_{1}>1$.

For this case, we have by $c^{\prime}(1)=1$, the strict convexity of $c$ and by the structure of the sharing rule, that the difference in Choquet expected utility for choosing the effort level of $s_{1}=1+\Delta$ instead of 1 is at most

$$
1+\Delta-c(1+\Delta)-1+c(1)=\Delta+c(1)-c(1+\Delta)<0,
$$

since we have $c(1+\Delta)-c(1)>\Delta$.

Therefore, we have that for $\Psi_{\widetilde{\nu}_{1}}\left([n-1, \rightarrow)_{\Delta}\right)<1-\frac{1}{\Delta} \cdot(c(1)-c(1+\Delta))$ the unique best response of agent 1 is $s_{1}=1$. 


\section{Proof of Theorem 5}

For each $j \in J$ let $\nu_{j}^{*}$ be a pure belief such that the ambiguity level $\Psi_{\nu_{j}^{*}}\left(\left\{\underline{\underline{L}}_{j}\right\}\right)>\frac{c(1)}{G+1}$. Then $s_{j}^{*}=1$ is the (unique) best response. By Lemma 7 , such $\nu_{j}^{*}$ exists.

For every pure belief $\nu_{1}^{*}$ with centre $1_{-1}$ some $s_{1}^{*}$ exists that maximizes

$$
\int^{C} f_{1}\left(\varphi\left(\underline{1}_{-1}, s_{1}\right)\right) d \nu_{1}-c\left(s_{1}\right)
$$

The combination $\left(\left(s_{k}^{*}\right)_{k \in N},\left(\nu_{k}^{*}\right)_{k \in N}\right)$ is an equilibrium in the game induced by the sharing rule.

Let $\left(s_{k}^{*}, \nu_{k}^{*}\right)$ be such an equilibrium.

Since $\Psi_{\nu_{j}^{*}}\left(\left\{\underline{0}_{-j}\right\}\right)>\frac{c(1)}{G+1}$, it follows from Lemma 7 that for each $j \in J$ we have $s_{j}^{*}=1$. Since $\left(s_{k}^{*}, \nu_{k}^{*}\right)$ is an equilibrium, $\nu_{1}^{*}$ is a pure belief at $\underline{1}_{-1}$. By assumption of the theorem, $\Psi_{\widetilde{\nu}_{1}^{*}}\left(\left[\widetilde{s}_{-1}, \rightarrow\right)_{\Delta}\right)<1-\frac{1}{\Delta} \cdot[c(1)-c(1-\Delta)]$, so it follows from Lemma 8 , that $s_{1}^{*}=1$. This completes the proof.

\section{Proof of Theorem 6}

According to Theorem 5 we have in equilibrium that $\forall k \in N: s_{k}^{*}=1$. So it remains to show that the Choquet expected utility for each agent $k \in N$ equals $1-c(1)$.

For agent 1, we have

$$
\left.\int^{C} f_{1}\left(\varphi\left(s_{-1}, 1\right)\right) d \nu_{1}-c(1)=1 \cdot\left[\widetilde{\nu}_{1}^{*}\left(\left[\widetilde{s}_{-1}^{*}, \rightarrow\right\rangle_{\Delta}\right)\right)-\nu_{1}^{*}(\emptyset)\right]-c(1)=1-c(1) .
$$

For any agent $j \in J$ we have

$$
\int^{C} f_{j}\left(\varphi\left(s_{-j}, 1\right)\right) d \nu_{j}-c(1)=1 \cdot\left[\nu_{j}^{*}\left(S_{-j}\right)-\nu_{j}^{*}(\emptyset)\right]-c(1)=1-c(1),
$$

which proves the theorem.

\section{Proof of Corollary 3}


Suppose $\forall k \in N: \widetilde{\nu}_{k}^{*}=\widetilde{\nu}^{*}$. For the equilibrium to be ex-post efficient, the assumption of Theorem 5 requires with respect to agent 1 that $\Psi_{\widetilde{\nu}^{*}}\left(\left[\widetilde{s}_{-1}, \rightarrow\right)_{\Delta}\right)<1-\frac{1}{\Delta} \cdot[c(1)-c(1-\Delta)]$. Since the belief is pure with centre $\underline{1}_{k}$, this is equivalent to $\widetilde{\nu}^{*}\left([n-1, \rightarrow)_{\Delta}\right)>\frac{1}{\Delta} \cdot[c(1)-c(1-\Delta)]$. For the agents in $J$, we must have $\Psi_{\nu^{*}}\left(\left\{_{-}\right\}\right)>\frac{c(1)}{G+1}$. Since the beliefs are pure with centre $\underline{1}_{-k}$, this holds whenever $\widetilde{\nu}^{*}\left((0, \rightarrow)_{\Delta}\right)<1-\frac{c(1)}{G+1}$.

Denote $a_{1}:=\widetilde{\nu}(\{n-1\})$ and $a_{2}:=\widetilde{\nu}\left([n-1, \rightarrow)_{\Delta}\right)$. Let $\widetilde{\nu}\left((0, \rightarrow)_{\Delta}\right)=a_{1}<1-\frac{c(1)}{G+1}$ and $a_{2}>\frac{1}{\Delta} \cdot[c(1)-c(1-\Delta)]$. Definition 1 requires that $a_{1}=\widetilde{\nu}(\{n-1\})<\widetilde{\nu}\left((0, \rightarrow)_{\Delta}\right)=a_{2}$. For $G$ sufficiently large, such $a_{1}$ and $a_{2}$ exist.

The pure belief

$$
\widetilde{\nu}(E):=\left\{\begin{array}{lll}
0 & \text { if } & n-1 \notin E \\
a_{1} & \text { if } & n-1 \in E \nsupseteq[n-1, \rightarrow)_{\Delta} \\
a_{2} & \text { if } & S \neq E \supseteq[n-1, \rightarrow)_{\Delta} \\
1 & \text { if } & E=S .
\end{array}\right.
$$

satisfies both conditions of Theorem 5 and is convex since

$$
\widetilde{\nu}\left([n-1, \rightarrow)_{\Delta}\right)+\widetilde{\nu}\left((0, \rightarrow)_{\Delta}\right) \leq \widetilde{\nu}(S)+\widetilde{\nu}(\{n-1\}) .
$$

